\title{
Effects of Cross Linking on the Chromatographic Nitrogen Isotope Separation
}

\author{
Xunyue Liu ${ }^{1}$, Xingcheng Ding ${ }^{1 *}$, Tatsuya Suzuki ${ }^{2}$, Masao Nomura ${ }^{2}$, Yasuhiko Fujii ${ }^{2}$ \\ ${ }^{1}$ Institute of Nuclear Agricultural Science, Zhejiang University, Hangzhou, China \\ ${ }^{2}$ Research Laboratory for Nuclear Reactors, Tokyo Institute of Technology, Tokyo, Japan \\ E-mail: dingxch@zju.edu.cn \\ Received November 15, 2010; revised January 26, 2011; accepted February 9, 2011
}

\begin{abstract}
The effects of cross linking with porous cation exchange resin were studied for nitrogen isotope separation. The displacement chromatography was conducted by the resin with cross linking rang from $20 \%$ to $40 \%$. A sharp adsorbed ammonium band was maintained for each operation. Enriched ${ }^{15} \mathrm{~N}$ isotopes with $0.93 \%$ were obtained by $20 \%$ cross linking resin and two meters chromatographic operation which started from the natural abundance of ammonium molecule. The effect of cross linking percentage on the height equivalent to a theoretical plate (HETP) was evaluated in the present work and the HETP value is proportional to the cross linking percentage. HETP value of $0.036 \mathrm{~cm}$ was obtained at the present system by using $20 \%$ cross linking resin.
\end{abstract}

Keywords: Cross Linking, Chromatography, Nitrogen Isotope, Ion Exchange Resin

\section{Introduction}

Enriched nitrogen isotope ${ }^{15} \mathrm{~N}$ has been used in various scientific and technological fields as a non-radioactive tracer [1]. In nuclear engineering, nitride fuel is the most appropriate fuel for the innovative nuclear reactors because of its outstanding characteristics such as high melting point, high thermal conductivity, high breeding ratio, etc. for fast breeder reactors (FBRs). FBRs with the nitride fuel of highly enriched in ${ }^{15} \mathrm{~N}$ isotope are considered as one of the promising directions for the development of safe atomic energy [2]. Natural nitrogen, however, cannot be used as the material of the nitride fuel, due to the neutron reactivity of the major isotope ${ }^{14} \mathrm{~N}$. The major isotope ${ }^{14} \mathrm{~N}$, is converted to the radioactive isotope ${ }^{14} \mathrm{C}$ in nuclear reactors whose half life is more than 5000 years by the reaction of ${ }^{14} \mathrm{~N}(\mathrm{n}, \mathrm{p}){ }^{14} \mathrm{C}$. The present urgent problem is lowering the price of nuclear fuel which is mainly affected by the large expense in ${ }^{15} \mathrm{~N}$ enrichment from the natural abundance of $0.365 \%$ up to the required 99.9\% [3]. For the sake of neutron economy and to avoid environmental risk of ${ }^{14} \mathrm{C}$, innovative technologies are needed for the production of highly ${ }^{15} \mathrm{~N}$ enriched nitrogen.

Nitrogen isotope separation by ion exchange chromatography has been studied since Urey et al. made ex- periments between gaseous ammonia and ammonium salts in aqueous solution using a cation exchange resin [4]. Separation and concentration of a stable isotope from an isotopic mixture with natural occurrence is a very complex problem and usually, the isotopic separation coefficients of natural abundance are very low. Recently, many studies have been focused on nitrogen isotope separation [5-10] and chemical exchange method in isotope separation had been proved to be the most promising method for light element isotope separation since firstly performed by Spedding et al. [11]. In this process, strong acidic cation exchange resin was packed in colums and ammonium ions were adsorbed in resin phase. Then, a $\mathrm{NaOH}$ solution was fed into the columns as an eluent. During this process, the light isotope ${ }^{14} \mathrm{~N}$ was enriched at the front boundary of ammonium ion adsorption band and the heavy isotope ${ }^{15} \mathrm{~N}$ was enriched at the rear boundary region. In general, cation exchange process is a promising technique to produce highly enriched isotope due to the nature of small HETP value. Among the operating parameters in cation exchange process, cross linking is know as a decisive factor on the process of chromatographic isotope separation. In this paper, as a continue work of our previous work, we studied nitrogen isotope separation by cation exchange resin with different percentage of cross linking to evalua- 
tion the chromatographic performance of ${ }^{15} \mathrm{~N}$ isotope enrichment.

\section{Experimental}

In the present work the experiments were performed by the cation exchange resin which was synthesized from the law material of styrene, the synthetic method was given in Figure $\mathbf{1}$ and Figure $\mathbf{2}$ was the microscope photographs of the synthesized resin. The detailed experimental conditions were shown in Table 1. The synthesized resins were packed uniformly in a jacketed glass column (1.0 m long, 8 mm I. D) and was preliminarily conditioned to $\mathrm{H}^{+}$form with $2.0 \mathrm{~mol} / \mathrm{dm}^{3} \mathrm{HCl}$ solution, then rinsed with pure water to remove free $\mathrm{H}^{+}$ions. One meter ammonium adsorption band was formed in the column by feeding $(0.2 \pm 0.1) \mathrm{mol} / \mathrm{dm}^{3} \mathrm{NH}_{4} \mathrm{OH}$ and was eluted by the eluent of ca. $(0.2 \pm 0.1) \mathrm{mol} / \mathrm{dm}^{3} \mathrm{NaOH}$ solutions which fed at the flow rate of $(1.0 \pm 0.1) \mathrm{ml} / \mathrm{min}$. The ammonium adsorption band moved down in the columns at the speed of $5.2 \mathrm{~m} /$ day. The thermostated water was circulated through the jacket to maintain the temperature at (308 \pm 1.0$) \mathrm{K}$ throughout the experiments.

The effluent out of the last column was collected in fractions by a fraction collector. Prior to the sampling, a certain volumes of $\mathrm{HCl}$ solutions were put into the sampling tubes to neutralize or acidify the sampled fractions. The sampled fractions of effluent were subjected to concentration analysis of ammonium ion by YokogawaHokusin IC-100 ion chromatography analyzer (Tokyo, Japan). Nitrogen isotopic abundance ratios of ${ }^{14} \mathrm{~N}$ and ${ }^{15} \mathrm{~N}$ in the samples were measured by two mass spectrometers ESCO EMD-05S double focusing mass spectrometer for gas samples and IsoPrime EA mass spectrometer, which accepts solid samples such as $\mathrm{NH}_{4} \mathrm{Cl}$. When ESCO EDM-05 was used, the samples collected in the chemical form of $\mathrm{NH}_{4} \mathrm{Cl}$ were converted to $\mathrm{N}_{2}$ samples by $\mathrm{KBrO}$ solutions prior to the analysis by mass spectrometry and the isotopic abundance of ${ }^{15} \mathrm{~N}$ was calculated from the ratio of the peak height.

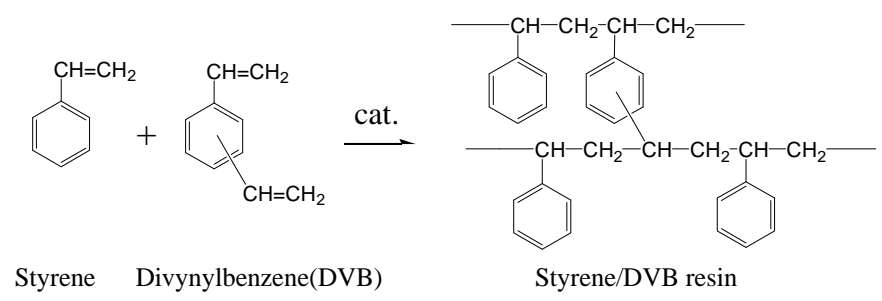

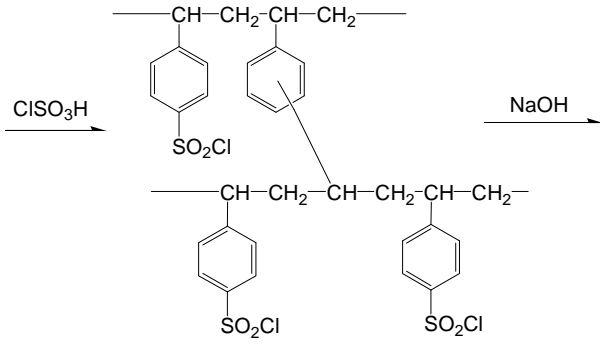

Chlorosulfonated resin

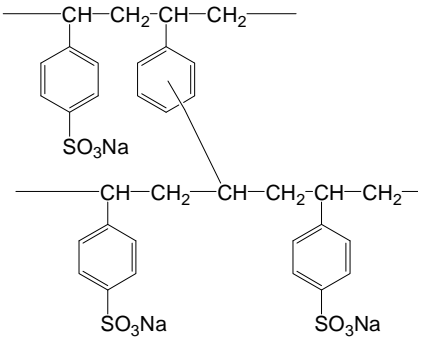

Ion-exchange resin

Figure 1. Synthetic method of the cation exchange resin.

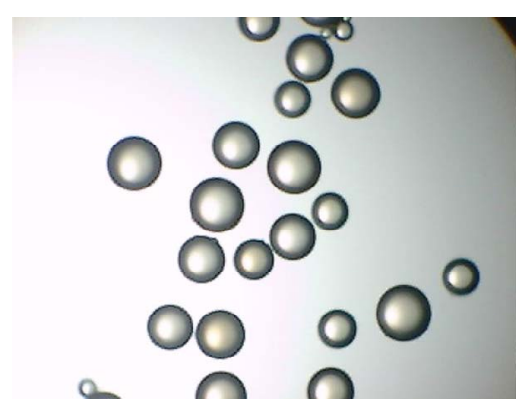

(a)

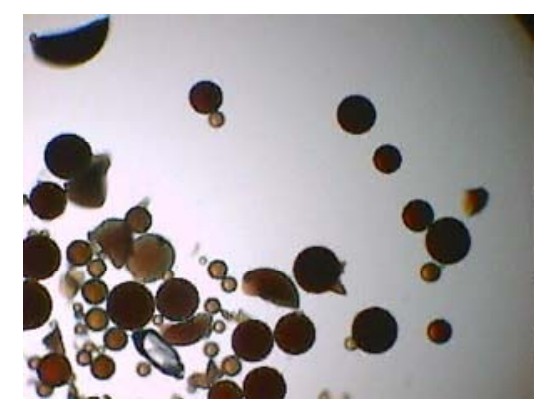

(b)

Figure 2. The microscope photographs of the synthesized resin: (a) Particle size: 60 - 100 mesh, cross linking 20\%; (b) Particle size: 100 - 250 mesh, cross linking $40 \%$. 
Table 1. Experimental conditions.

\begin{tabular}{l}
\hline Cation exchange resin: Strongly acidic type, cross linking $20 \%-40 \%$, particle size $60-250$ mesh \\
\hline Feed solution: $(0.2 \pm 0.1) \mathrm{mol} / \mathrm{dm}^{3} \mathrm{NH}_{4} \mathrm{OH}$ \\
Effluent solution: $(0.2 \pm 0.1) \mathrm{mol} / \mathrm{dm}^{3} \mathrm{NaOH}$ \\
Column diameter: $8 \mathrm{~mm}$ \\
Flow rate: $(1.0 \pm 0.1) \mathrm{mL} / \mathrm{min}$ \\
Migration distance: $(2.0 \pm 0.05) \mathrm{m}$ \\
Band velocity: $(5.2 \pm 0.2) \mathrm{m} / \mathrm{d}$ \\
Temperature: $(308 \pm 1.0) \mathrm{K}$
\end{tabular}

\section{Results and Discussion}

Three different percent cross linking resins were performed for nitrogen isotope separation. The chromatographic concentration curve and isotopic enrichment profile of $20 \%$ cross linking resin as an example was given in Figure 3. It could be seen in Figure that the profiles of ammonium ion bands were very sharp at the boundaries and ${ }^{15} \mathrm{~N}$ was apparently enriched at the rear boundary. It is necessary to maintain the sharp ammonium band in order to obtain highly enriched ${ }^{15} \mathrm{~N}$; the results clearly indicate that the present chromatographic operation conditions were suitable for nitrogen isotope separation. Since the chromatographic operations were conducted in a reverse breakthrough manner, the isotopic abundance ratios in the middle band fractions of the chromatogram were equal to the isotopic abundance in the original feed solution. This means no remixing occurred in the middle band during the present operation.

When different cross linking was synthesized, high cross linking means high divynylbenzene (DVB) materials contained in the resin structure and this means the percentage of ion exchange functional group was decreased in the same amount resin. Figure $\mathbf{4}$ was the relationship between ${ }^{15} \mathrm{~N}$ enrichment percentage and cross linking. Enriched ${ }^{15} \mathrm{~N}$ isotopes were decreased from 0.93 to 0.68 when compared with the cross linking of twenty and forty percentage. Since high cross linking means low exchange capacity, it is reasonable that the ${ }^{15} \mathrm{~N}$ enrichment percentage is reduced with the increase of cross linking at the same given migration distances. SQS-6 resin was the commercial resin and with $8 \%$ cross linking which used in References [8-10], ${ }^{15} \mathrm{~N}$ enrichment percentage was $1.56 \%$ when SQS-6 resin was performed under the same condition, and low cross linking resin has much higher enrichment ability than high cross linking resin.

The separation of nitrogen isotopes by means of cation exchange resin is based on the isotopic fractionation between ammonia in aqueous solution and ammonium ion in the ion exchange resin as shown below:

$$
\mathrm{R}^{14} \mathrm{NH}_{4}{ }^{+}+{ }^{15} \mathrm{NH}_{3}=\mathrm{R}^{15} \mathrm{NH}_{4}{ }^{+}+{ }^{14} \mathrm{NH}_{3}
$$

where $\mathrm{R}$ represents the fixed anion in the resin.

In order to evaluate the performance of the ${ }^{15} \mathrm{~N}$ enrichment in displacement chromatography, the height equivalent to a theoretical plate (HETP) is introduced. In a transient state, as in the case of the present work, HETP is usually obtained by a computer calculation fitting
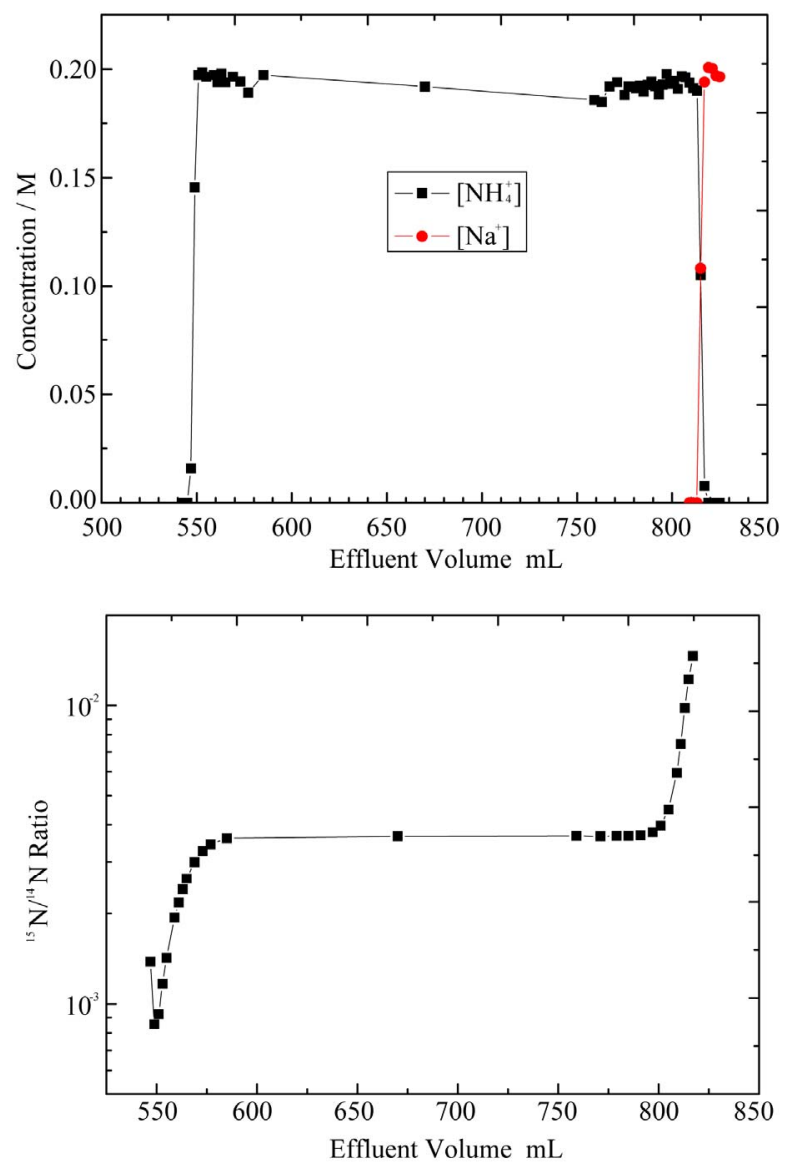

Figure 3. Chromatographic concentration curve and isotopic enrichment profile of $20 \%$ cross linking resin. 


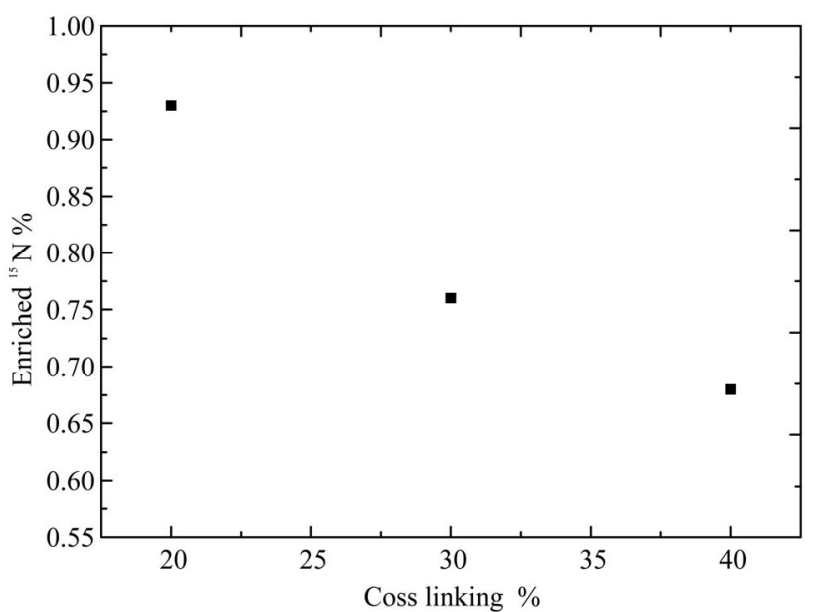

Figure 4. Relationship between enriched ${ }^{15} \mathrm{~N}$ and cross linking.

based on a cascade theory. However, Fujii et al. proposed more convenient equations to analyze chromatographic data on isotope separation [12]. These equations are used in the present work and the HETP is determined by the following equation for the transient state isotope separation:

$$
H=\frac{\varepsilon}{k}\left\{1+\frac{R o}{\exp (\varepsilon k R o L)-1}\right\}
$$

When the enrichment extent is not so large, i.e. $\varepsilon k R o L \ll 1$,

$$
H=\frac{\varepsilon}{k}+\frac{1}{k^{2} L}
$$

where $H$ is the HETP, the separation coefficient, $k$ is the slope coefficient, $R o$ is the original isotope atomic fraction, $L$ is the migration length.

The observed HETP of different cross linking was plotted in Figure 5. It is seen in the figure that cross linking can affect the HETP value obviously. HETP value proportionally increase with the cross linking at the present work and low cross linking has much advantage for HETP. HETP value of $0.036 \mathrm{~cm}$ was obtained at the present system by using $20 \%$ cross linking resin. Previously, Aida et al. reported the boron isotope separation by using commercially available anion exchange resin (Diaion WA-21, 60 - 80 mesh) and got the value of HETP was $0.18 \mathrm{~cm}$ [13]. Ohwaki et al. reported nitrogen isotope separation by using HITEC-H1 cation exchange resin (porous strong acid type, cross linking 30\%, particle size 37 - $77 \mathrm{um}$ ) and the value of HETP was $0.019 \mathrm{~cm}$ [7,14]. In our previous work [8-10], SQS-6 resin (8\% cross linking, particle size $60-80$ um) was applied to nitrogen isotope separation and the value of HETP was $0.02 \mathrm{~cm}$. Comparing with above mentioned HITEC-H1

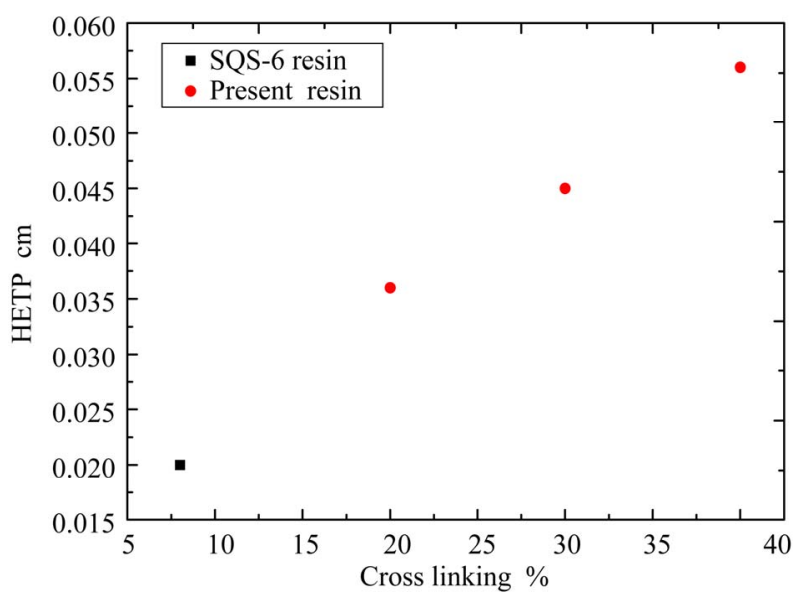

Figure 5. Observed HETP values and cross linking (Note: SQS-6 was the different type resin which used by our previously experiments, its detail information can see the References [7-10]).

and SQS-6 resin, the present resin has quite large HETPs. Sugiyama and his co-worker studied nitrogen isotope separation by using cryptand resin [15], the HETP value was evaluated $(0.5 \pm 0.2) \mathrm{cm}$. The present resin has much advantage than that cryptand resin. HETP values are small in the present operation when the enrichment of ${ }^{15} \mathrm{~N}$ is steadily proceeding and the present resin can be used for the nitrogen isotope separation.

\section{Conclusions}

Chromatographic nitrogen isotope separation was conducted by using cation exchange resin with different cross linking percentage cation exchange resin. It was found that a sharp boundary was obtained for each different cross linking chromatographic operation and it is important for nitrogen isotope enrichment. With the same migration distance, low cross linking resin has the large nitrogen isotope enrichment capacity. The values of HETP of the present study have shown increase with high cross linking, the present $20 \%$ cross linking resin has quite large HETP value compare with other type of cation exchange resins but much smaller than the cryptand resin in Reference [15].

\section{Acknowledgements}

The present paper was partially financially supported by the Special Fund for Agro-scientific Research in the Public Interest (201103007).

\section{References}

[1] S. J. Adelstein and F. J. Manning, “Isotopes for Medicine 
and Life Sciences,” National Academy Press, Washington, D.C., 1995.

[2] V. D. Borisevich, O. E. Morozov, Yu. P. Zaozerskiy, G. M. Shmelev and Y. D. Shipilov, "On the Enrichment of Low-Abundant Isotopes of Light Chemical Element by Gas Centrifuges," Nuclear Instruments and Methods in Physics Research Section A: Accelerators, Spectrometers, Detectors and Associated Equipment, Vol. 450, No. 2-3, 2000, pp.515-521. doi:10.1016/S0168-9002(00)00266-7

[3] E. Aoki, T. Kai and Y. Fujii, "Theoretical Analysis of Separating Nitrogen Isotopes by Ion-Exchange,” Proceedings of the 5th Workshop on Separation Phenomena in Liquids and Gases, Iguazu Fals, 22-26 September 1996, p. 197.

[4] H. C. Urey, J. R. Huffman, H. G. Thode and M. Fox, “Concentration of ${ }^{15} \mathrm{~N}$ by Chemical Methods," Journal of Chemical Physics, Vol. 5, No. 11, 1937, pp. 856-869. doi:10.1063/1.1749954

[5] W. K. Park and E. D. Michaels, "Separation of Nitrogen Isotopes by Displacement Band Chromatography," Separation Science and Technology, Vol. 23, No. 12-13, 1988, pp. 1875-1889. doi:10.1080/01496398808075669

[6] A. V. Kruglov, B. M. Andreev and Y. E. Pojidaev, "Continuous Isotope Separation in Systems with Solid. II. Separation of Nitrogen Isotopes with Use of Ion-Exchange Resin," Separation Science and Technology, Vol. 31, No. 4, 1996, pp. 471-490. doi:10.1080/01496399608002211

[7] M. Ohwaki, Y. Fujii and M. Hasegawa, "Flow-Rate Dependence of the Height Equivalent to a Theoretical Plate in Nitrogen Isotopes Separation by Displacement Chromatography," Journal of Chromatography A, Vol. 793, No. 2, 1998, pp. 223-230. doi:10.1016/S0021-9673(97)00941-2

[8] X. Ding, T. Suzuki, M. Nomura, A. Aida and Y. Fujii, "Nitrogen Isotopes Enrichment for Nitride Fuel by Using
Hybrid Chemical Exchange Process,” Progress in $\mathrm{Nu}$ clear Energy, Vol. 47, No. 1-4, 2005, pp. 420-425. doi:10.1016/j.pnucene.2005.05.042

[9] X. Ding, T. Kaneshiki, M. Nomura and Y. Fujii, "High Enrichment of ${ }^{15} \mathrm{~N}$ Isotope by Ion Exchange for Nitride Fuel Development,” Progress in Nuclear Energy, Vol. 50, No. 2-6, 2008, pp. 504-509. doi:10.1016/j.pnucene.2007.11.070

[10] X. Ding, M.Nomura, T. Suzuki and Y. Fujii, "High Enrichment of ${ }^{15} \mathrm{~N}$ by Chromatographic Chemical Process," Journal of Chromatography A, Vol. 1201, No. 1, 2008, pp. 65-68. doi:10.1016/j.chroma.2008.06.023

[11] F. H. Spedding, J. E. Powell and H. J. Svec, “A Laboratory Method for Separating Nitrogen Isotopes by Ion Exchange," Journal of the American Chemical Society, Vol. 77, No. 23, 1955, pp. 6125-6132. doi:10.1021/ja01628a010

[12] Y. Fujii, M. Aida, M. Okamoto and T. Oi, “A Theoretical Study of Isotope Separation by Displacement Chromatography," Separation Science and Technology, Vol. 20, No. 5-6, 1985, pp. 377-392. doi:10.1080/01496398508060688

[13] M. Aida, Y. Fujii and M.Okmoto, "Chromatographic Enrichment of ${ }^{10} \mathrm{~B}$ by Using Weak-Base Anion-Exchange Resin,” Separation Science and Technology, Vol. 21, No. 6-7, 1986, pp. 643-654. doi:10.1080/01496398608056140

[14] M. Ohwaki, Y. Fujii, K. Morita and K. Takeda, "Nitrogen Isotope Separation Using Porous Microreticular Cation-Exchange Resin,” Separation Science and Technology, Vol. 33, No. 1, 1986, pp. 19-31. doi:10.1080/01496399808544753

[15] H. Sugiyama, Y. Enokida and I. Yamamoto, “Nitrogen Isotope Separation with Displacement Chromatography Using Cryptand Polymer," Journal of Nuclear Science and Technology, Vol. 39, No. 4, 2002, pp. 442-446. doi:10.3327/jnst.39.442 